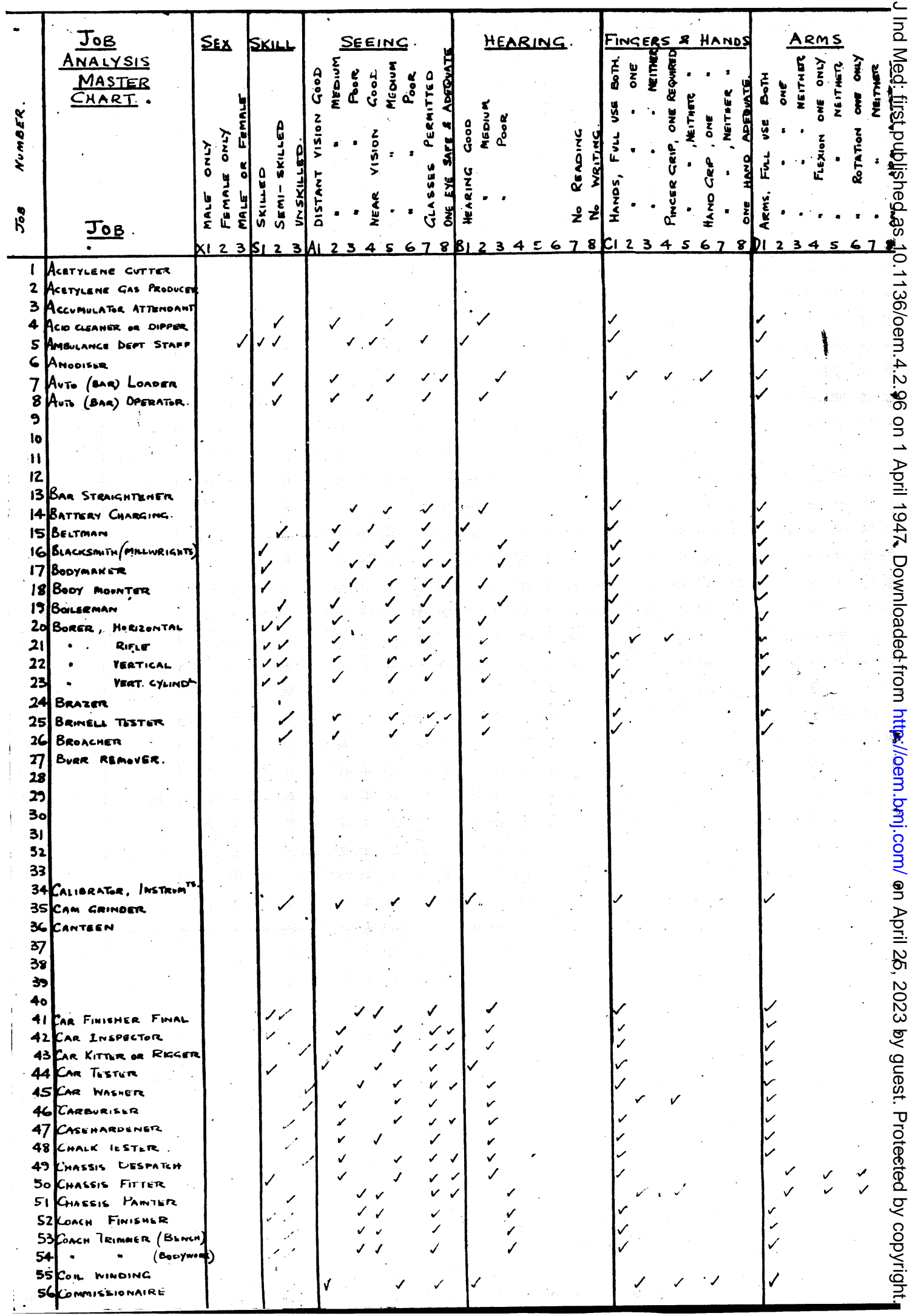




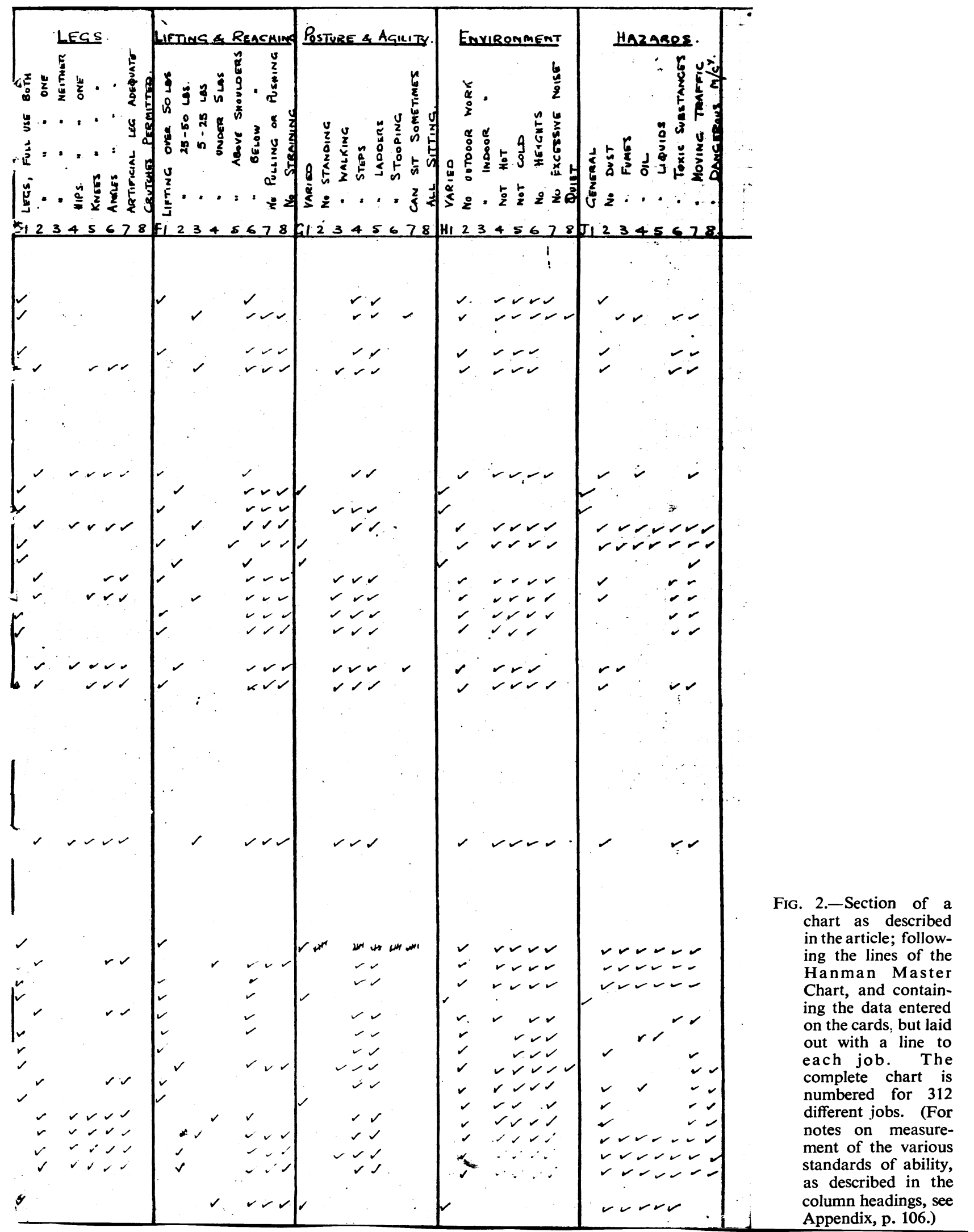


Brit. J. industr. Med., 1947, 4, 102.

\title{
THE PLACEMENT IN INDUSTRY OF THE PHYSICALLY HANDICA PPED \\ A PRACTICAL METHOD OF LINKING THE DISABLED PERSON WITH SUITABLE OCCUPATIONS
}

\author{
BY \\ N. L. LLOYD \\ From Rolls-Royce, Ltd., Derby
}

(RECEIVED FOR PUBLICATION, OCTOBER 2, 1946)

The problem of the placing of disabled persons in industry, which was of such importance during the war years, has not disappeared on return to peacetime conditions, and the Disabled Persons (Employment) Act, 1944, turns the moral obligation of the employer into a duty. National schemes for the registration of such individuals may be designed; industry may be exhorted, cajoled, or directed to employ this or that proportion of handicapped workers; the Ministry of Labour and National Service may plan the problem centrally and through Employment Exchanges; but the ultimate success of the idea and its practical fulfilment will finally depend on the willing co-operation of each individual industry, of each individual firm, and of each individual labour bureau in that firm.

Great efforts were made under the stress of war to absorb disabled persons into the war production army, and the methods of placing those so handicapped have varied from simple to elaborate schemes. These methods are still in the stage of evolution, and, in our search for the ideal plan, schemes are being developed in some industrial units which have long since been discarded by others as too time-consuming or otherwise impractical. A pooling of knowledge and experience is necessary.

In general the problem has hitherto been dealt with by one of three methods. The most primitive may be called the 'trial and error' method. A man is tried on a job, and if he is found incapable he may be tried on another, and so on until he is marked unemployable as far as the factory is concerned. In the 'personal memory' method, the labour manager carries in his head a list of jobs which are suitable for various disabilities; one-armed men, one-legged men, and so on. A labour manager of experience may, indeed, have a very complete picture of such jobs in his mind, but dependence on individual memory has the great disadvantage that when the labour manager leaves, or is away temporarily, his memory is no longer available; the department is thus at a loss, and the company he serves thereby suffers. The third method may be called the 'disability list' method. The labour manager's memory is replaced by lists of jobs which can be done by workers with certain disabilities. This is an advance on the first two methods, but usually results in lists containing a few 'odd-lot' jobs of a dead-end variety, including such jobs as lavatory attendant, weighbridge keeper, or night watchman.

The method here described may be called the " job analysis" method. It has been developed during the recent war, particularly in the British Army and in the United States of America.

\section{Job Analysis}

I believe it to be essential for the proper absorption and utilization of disabled persons that there should be an ordered scheme of analysis of jobs which will indicate the physical characteristics that each job demands from its operator. Many such schemes have been designed, some simple, some intricate. One of the simpler is the Pulhems system, which is used in the British Army and elsewhere. Jobs and men are assessed in seven main characteristics (the initials of which give the name to the system-physique, upper limb, locomotion, hearing, eyesight, mental ability, and stability (emotional). Some other methods are most elaborate and complicated. Most of them 'work,' but this is not enough. The successful system is one which not only works but is in fact used. Many industrial medical officers and personnel managers are concerned with this problem, and some have evolved their own particular way of tackling the matter, the object of which is to match the man to the job to the mutual advantage of the man and the industry.

This paper presents a scheme which is more eleborate than that used in the Services, the Pulhems . system appearing inadequate for modern industrial purposes. It is not so elaborate as some American 
schemes, which appear too complicated for practical everyday use, but which nevertheless have been found to be helpful in certain respects. The best of each has been used to make up the scheme now presented, which is not put forward with any claim to originality, but is rather the result of an abstraction in the light of my own experience of what has appeared to be useful in a number of schemes.

A form was designed for recording the minimum physical requirements of each job. It will be seen that the form (see legend to fig. 1) lists nine groups of physical characteristics, environmental conditions, and hazards, with usually eight subdivisions in each group; after each sub-division there is a small ruled-off square. This form is used in the first step in job analysis, and a tick is placed in the small square corresponding to that degree of physical capacity which represents the minimal requirement for the job, or which indicates the demand on physical effort, postural ability, etc., or, in the case of hazards, the absence of hazards which might prevent a particular man from undertaking the job. While there may be an inclination to record other physical attributes on this form-and in some industries they may be called for-a warning is given against over-elaboration, leading to unwieldiness, which will defeat the object of the system, namely, rapid job placement. However, each group has a spare line, and there are three spare groups, at K 1-9, L 1-9, and M 1-9 for future additional entries should these become necessary.

It should perhaps be stressed that the completion of this form should not be a matter of guess-work, or of probabilities, or of vague knowledge. It is not a fireside occupation. It will be found in practice that the only satisfactory way of completing the required details is the three-step method of (1) seeing the job done, (2) talking to the man who does it, and (3) talking to the superintendent of the department where the job is done. It will soon be found that the word 'job' conveys a meaning which is different from that usually understood in the labour bureau, and that many jobs on the Labour Code represent a number of varieties of work which cannot be satisfactorily analysed except by subdividing the main job into a number of sub-headings. The job of labourer is perhaps as good an example as any; there are general labourers, stores labourers, millwrights' labourers, laboratory labourers, etc., whose jobs each differ from each other in their physical requirements.

Although the assessment of jobs in this manner is not one which is necessarily best done by the industrial medical officer, he should, nevertheless, give help concerning the standards to be used. Indeed, co-operation is essential, for it is important that the assessor and the medical officer should talk the same language. The meaning of good, medium, etc., as applied to vision must be clearly understood; a number of headings, necessarily brief on the form, require explanation or elaboration. I have attached my own personal meanings to these headings and shall refer to them at the end of the paper. It is sufficient to say that the co-operation between medical officer, assessor, and personnel manager should be such that when the medical officer gives his opinion that an employee is fit to work with certain limitations, he can be confident that, guided by the assessor's analysis, the right kind of job will be found. For this reason it may be as well that medical officers should have a considerable hand in analysis, or at least in the training of assessors. Analysis actually undertaken by the medical officer will be invaluable to him, though pressure of work may make it difficult.

It is important that the analysis should be agreed on by the superintendent of the department where the job is located, since he may in the future be asked to employ a man with a disability and his co-operation will be essential. His prior agreement is obviously of great value. It is also important that the medical officer, in his pre-employment medical examination, should think along the lines of this analysis and express his conclusions in such a way that they conform to the information available on the analysis sheets. Physical diseases and deformities must be translated into abilities and disabilities as shown on the form (fig. 1).

\section{Matching the Man and the Job}

The next part of the problem is to match the disabled person with the suitable job. Is there a rapid, simple, and practical method by which jobs within the capacity of handicapped men can be brought up for consideration? The scheme must indicate jobs which are within the capacity of individuals with various combinations of handicaps, and it must enable the personnel manager to say with confidence whether or not a suitable job is available for a particular case.

After consideration of a number of mechanical and other methods of sorting, it was decided to put the information obtained from the job analysis on to cards (The Paramount Card, obtainable from CopelandChatterson Co., Ltd., Stroud, Gloucestershire). These cards have holes punched round the edges at the top and bottom and both sides; a hole can be converted into a ' $V$ ' with a simple instrument; each hole can be labelled to correspond with a particular item of information, and is to be cut into $a$ ' $V$ ' when that information applies. When the cards are properly aligned, and a finder passed through a particular hole and raised, intact cards will be lifted up while those in which the hole has been cut into a ' $V$ ' fall out. Such a card has been designed for the present purpose, the information being recorded as on the analysis sheet. Holes have been allotted to each physical demand, and, where a particular characteristic is ticked as being applicable, the corresponding hole is converted into a ' $\mathrm{V}$.'

When an applicant for work is found on medical examination to have a disability which limits the full range of normal activities in any way, the possible jobs which it would be wise and safe for him to undertake can quickly be found by inserting the finder in the appro- 


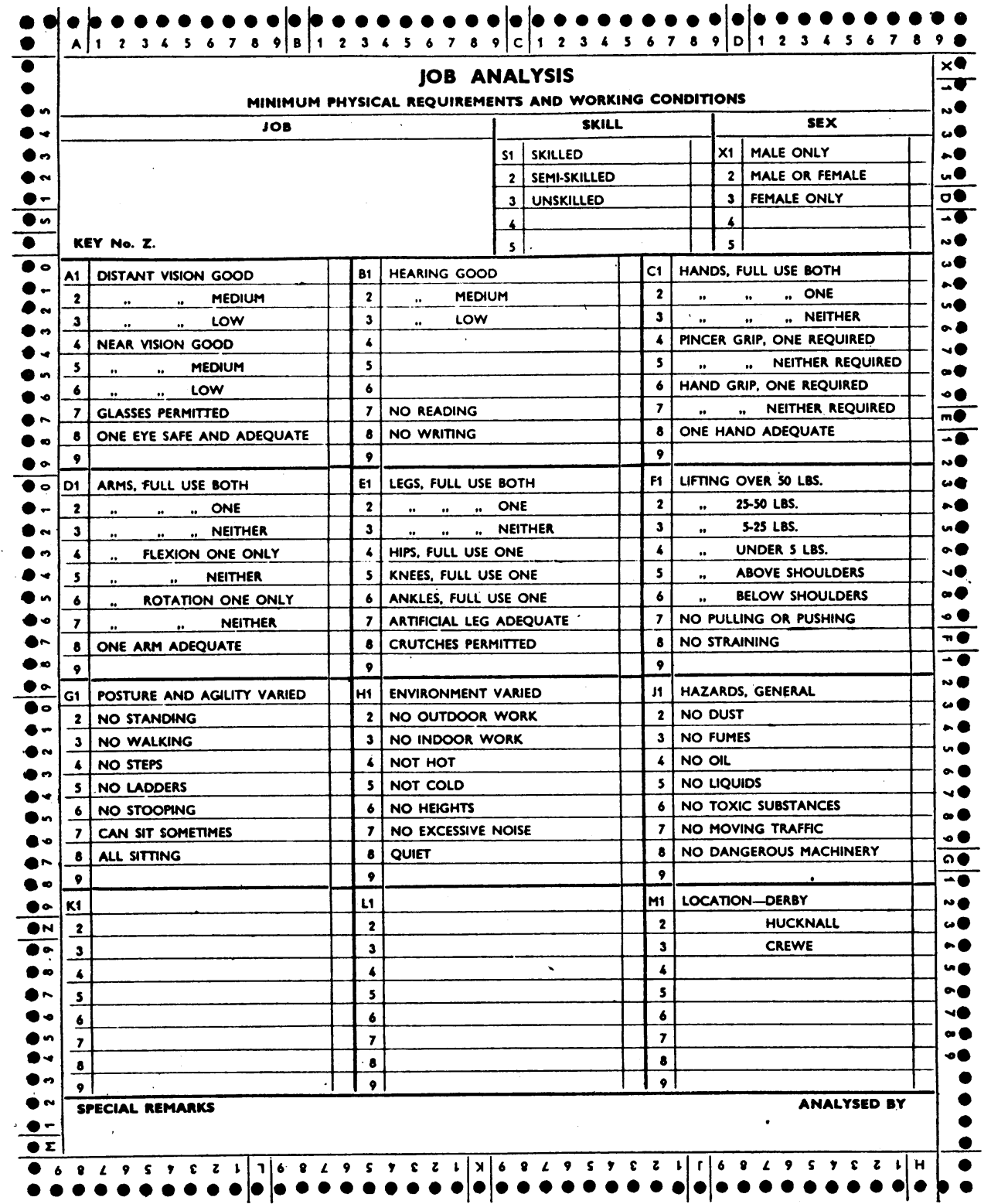

FIG. 1.-Card for recording minimum physical requirements of particular jobs. The form spoken of at p. 103 is made out exactly like the card, except that the punched holes with their corresponding letters and numbers are absent. (For notes on the standards used, and the interpretation of headings on this card, see Appendix, p. 106.)

(NoTE.-Section D had two sets of punched holes erroneously printed for it. This is a mistake that will be remedied in future printings.) 
priate hole and raising the cards on the finder; those cards relating to jobs to which that particular disability is no bar will fall out and plainly bring to notice all the jobs within his physical capacity. In cases where two disabilities or limitations apply, the finder will be passed through twice, or, alternatively, two finders may be used simultaneously. It does not matter in what order the cards are assembled, and it is not necessary to put them back in order.

The choice of suitable employment is limited by the matter of skill, a number of jobs being reserved for skilled men. It would be a waste of time for the labour bureau to search through skilled jobs where the applicant is, unskilled, and the cards have, therefore, been marked according to whether the job is skilled, semi-skilled, or unskilled, and appropriate holes allotted. In practice, the labour bureau will keep its cards in several 'packs,' separating the skilled from the other jobs, the male from the female, etc.

For the industrial medical officer who is not wholly familiar with all the varying occupations in the factories with which he is concerned, the cards will prove of value when a man is referred for an opinion as to fitness for a particular job. By referring to the appropriate job analysis card the medical officer can learn quickly the various physical attributes which are essential and those which are unnecessary. In order to find a particular card, the cards have been numbered and thirty holes set aside, numbering 0 to 9 three times, thus making it possible to find with ease a card with any number from 0 to 999.

In some circumstances the indication of the "location' of the job will be helpful, particularly where there are a number of separate factories with a central labour bureau. It is not uncommon to find that a job called by a particular name in one factory requires different characteristics from that called by the same name in another factory, even within the same organization. An obvious example is where one factory is built entirely on ground level and another on two or more floors. In the latter some occupations may require the use of steps, which are non-existent in the former. It may, therefore, be as well to prepare two cards, identical except for the reference to 'steps ' and to 'location.'

For some purposes it is more useful to have the information given on the cards available in the form of a chart. The idea of the chart was given to me, in a personal communication, by Mr. Hanman, Chief of the Physical Demands and Incapacities Analysis Section of the District Civilian Personnel Office, Twelfth Naval District, San Francisco, whose contribution is full of much thought and constructive criticism. The chart illustrated in fig. 2, pp. 96-97, following the lines of the Hanman Master Chart, contains the data to be found on the cards, but is laid out with a line to each job. The value of the chart lies chiefly in providing quick reference to the characteristics required by a particular job, thus saving the abstraction of the particular card from the pack. The Hanman chart is, however, used for more than this. It can take the place of the perforated cards here described in direct matching up of man to job. Neverthe- less, for the purpose of discovering quickly all suitable jobs for a particular disabled man, I believe that the Paramount card is more suitable and more likely to be used.

\section{Summary}

A scheme is described by which a disabled man can be matched to a job. There are both limitations and disadvantages in the scheme, which is regarded as being in the experimental stage. It covers only a few of the problems demanding attention and which call for more investigation. A valuable contribution will be made to the solution of the problems of the Ministry of Labour and National Service if combined information on these lines from many sources and from large numbers and varieties of industries is made available.

The investigation represents field work which must be done at the periphery, but the results should be centralized and made available to others so that ground covered need not be covered again.

A plea is put forward for the extensive use of job analysis, from the point of view of the physical demands made by each job, as a necessary preliminary to the satisfactory absorption in industry of the physically handicapped individual.

A practical method of recording these demands, physical and environmental, is described.

A new method is described of linking the disabled individual with the job shown to be suitable by analysis.

I am indebted to a number of individuals who have helped me in this problem. In particular I would mention Dr. R. S. F. Schilling Secretary of the Industrial Health Research Board of the Medical Research Council, Mr. Bert Hanman, War Manpower Commission, San Francisco, Mr. H. C. Weston, Senior Investigator, The Industrial Health Research Board, and Mrs. Bridget Bramwell, late of the Health Research The U.S. Office of War Information, The American American Library, The thanks are due also to a large number of the Itaff and Employees of Rolls-Royce, Ltd., who have assisted me in Staff and Employees of Rolls-Royce, Ltd., who have assisted me in analysing jobs or have supplied constructive criticism, and to Mr. T. S Haldenby, General Manager, Rolls-Royce, Ltd., for encouragement in this work and for permission to publish this article. The list of consulted and that have contributed to the final scheme.

\section{REFERENCES}

Duncan, G. W., Hanley, H. G., and Morton, H. J. V. (1945). Lancet, $2,379$.

Griffiths, H. E. (1935). 'Injury and Incapacity.' London.

Hanman, Bert (1945). Industrial Medicine, 14, 405.

Occupational Rating Plan for Manual and Shop Occupations. Attribute Handbook (undated). The Industrial Management Society. Chicago.

Operations Manual for Placement of the Physically Handicapped (1944). U.S. 3rd edit. 766 .

Patterson, J. W. T. (1945). Lancet, 2,

Putting the Disabled Veteran back to Work (1945). Industrial Hygiene Foundation of America, Pittsburgh. Proceedings of Hygiene Foundation of America, Pittsburgh.

Training and Reference Manual for Job Analysis (1944). War Manpower Commission, Bureau of Manpower Utilization. Mashington.

Utilization of Persons having Physical Limitations (1942). Division of Occupational Analysis and Manning Tables, War Manpower Commission, Bureau of Manpower Utilization. Washington. 


\section{APPENDIX}

NOTES ON THE STANDARDS USED AND THE INTERPRETATION OF OTHER HEADINGS ON JOB ANALYSIS SHEETS AND CARDS

In using the analysis sheets and cards, the following standards and interpretations were understood, so that there should be adequate co-operation between medical officers and personnel managers. It was necessary to have these clearly determined since there are no agreed standards in common use in industry.

\section{Vision}

Distant vision good:

Ability to read with each eye separately the sixth line of the Snellen test type card at a distance of twenty feet. (V.A. 6/9 6/9 or better.)

Distant vision medium:

Ability to read the fourth line of the Snellen test type card at twenty feet with each eye separately. (V.A. $6 / 12$ to $6 / 18$.) This standard is the minimum allowed for drivers of motor vehicles on the public highway.

Distant vision low:

Less than the above standards.

Near vision good:

Ability to read with each eye separately at a distance of thirteen inches the Jaeger test types Nos. 1 or 2.

Near vision medium:

Ability to read as above Jaeger test types Nos. 3 to 6.

Near vision low:

Less than the above.

In assessing the job the degree of vision required will depend on the two factors of safety and adequacy. Very few factory jobs require distant vision of any kind for the actual performance of the job, but there are a number which are done under circumstances carrying a risk of injury from moving traffic, overhead cranes, etc., calling for sudden avoiding movement. Such jobs, from the safety aspect, 'will call for medium distant vision.

When the vision of one eye is in the "low' category, the individual should for job placement purposes be classed as one-eyed in the category of the better eye, and should be employed only on work where it is considered that one eye is safe and adequate.

\section{Hearing}

Hearing good:

Ability to hear whispered speech at ten feet with each ear separately.

Hearing medium:

Ability to hear conversational speech at ten feet, with both ears together.

Hearing low:

Less than the above.

\section{Use of Limbs}

Full use of hands, arms, and legs:

From the assessor's point of view this means exactly what it says. From the medical officer's point of view it is important that function is con- sidered rather than anatomical perfection. Many a workman with a missing forefinger finds this no handicap whatever.

Lifting:

This refers to lifting without help. The medical officer will consider under this heading the state of the man's hands, arms, and back, and the condition of his heart, presence of a rupture, etc.

\section{Environment}

Heat and cold:

Temperatures over $80^{\circ} \mathrm{F}$. are to be considered hot; those below $55^{\circ}$ are to be considered cold.

Heights:

Apart from work on roofs and ladders, there are a few jobs where a man stands on a platform. The criterion here is whether a slip or fall is likely to cause more injury than a slip or fall when standing on ground level. From the medical aspect a liability to giddiness would call for a job with 'no heights'.

Loud noise:

This means something more than the average shop noise-for example, the noise of test-beds, tinsmiths, drop forge, or pneumatic drills.

Quiet:

This refers to jobs of the degree of quietness of an office.

\section{Hazards}

Dust fumes:

This means no more than the average factory dust or fumes, which are, in fact, very little. Most jobs fall into this category.

Oil:

This has particular reference to the risk of oil dermatitis, and, therefore, not only contact with cutting oils on machines, but the handling of oily components would be excluded here.

Toxic substances:

This refers to all those substances which may have an ill-effect on health on reaching the interior of the body, whether absorbed through the gastrointestinal tract, the lungs, or the skin. It does not include substance liable to cause dermatitis or ulceration of the skin.

Dangerous machinery:

All machine tools are potentially dangerous. In addition this heading can conveniently be used to include other dangers, such as pits, tanks, etc., into which a man might fall. This heading refers to stationary machinery.

Moving traffic:

Not only power-driven trucks and other vehicles are referred to here; there are also included overhead cranes and other hazards which might call for sudden avoiding movement. The medical officer will consider visual acuity and hearing and also the workman's agility on his legs. Workmen with artificial legs'will be better away from such risks. 